\title{
Risiko Nachtdienst
}

\section{Das größte Gesundheitsproblem ist die Überlastung}

\author{
Krebsgefahr durch Nachtdienste - eine aktuelle Studie beunruhigt \\ Pflegekräfte, die regelmäßig nachts arbeiten. Wird genug getan, um \\ sie vor gesundheitlichen Folgen des Schichtdienstes zu schützen? \\ Wir fragten nach bei Johanna Knüppel vom Deutschen Berufsverband \\ für Pflegeberufe (DBfK). Seit vielen Jahren beschäftigt sie sich mit dem \\ Arbeitsschutz in Pflegeberufen.
}

? Frau Knüppel, einer aktuellen Studie zufolge erhöhen längerfristige Nachtdienste bei Pflegerinnen das Risiko, an Krebs zu erkranken. Wie ordnen Sie diese Ergebnisse ein?

Knüppel: Ein erhöhtes Brustkrebsrisiko ist für Frauen mit Nachtschichten schon seit Jahren im Gespräch. Allerdings waren die Studien aus Dänemark, die das vermeintlich belegten, methodisch umstritten und die Aussage wissenschaftlich nicht haltbar. Auch die jetzt bekannt gewordenen Ergebnisse eines internationalen Review zeigen erhebliche Schwächen. Es ist die Rede von einem erhöhten Risiko, an Krebs der Brust, der Lunge, des Gastrointestinaltrakts oder der Haut zu erkranken. Man sucht aber vergeblich nach Aussagen, ob die Erkrankten z.B. Raucherinnen waren, wie sie sich ernährten, welches Freizeitverhalten sie hatten, wie intensiv sie Nachtschichten geleistet hatten bis zur Erkrankung.

Welche Faktoren lösen tatsächlich diese Krebsarten aus und gibt es einen unmittelbaren Zusammenhang mit Nachtschichten? Hier wären weitere Fragen zu stellen wie: Ernähren sich Menschen, die Nachtschichten leisten, ungesünder als Personen ohne Nachtarbeit? Sind sie stärkere Raucherinnen? Die Empfehlungen für eine möglichst risikoarme Gestaltung von Nachtdiensten sind alle bekannt: nur wenige hintereinander, ein verlässlicher Dienstplan, im Anschluss genügend ungestörtes Frei, damit die Umstellung gut gelingt und so weiter. Die gelten jetzt natürlich erst recht.
? Schichtdienste sind in der Pflege unvermeidbar. Wird genug getan, um die Mitarbeiter vor negativen gesundheitlichen Auswirkungen zu schützen?

Knüppel: Die negativen gesundheitlichen Auswirkungen bei Beschäftigten im Nachtdienst resultieren vor allem aus der hohen Arbeitsverdichtung. Die Nachtdienststudien der Pflegewissenschaftler aus WittenHerdecke und die Erhebung von Verdi haben sowohl für die Krankenhäuser wie für die stationäre Altenhilfe katastrophale Personalschlüssel im Nachtdienst ergeben. Mehr als 50 und zum großen Teil schwer pflegebedürftige Bewohner muss die Nachtwache in vielen Häusern betreuen. In der Regel gibt es keine Ablösung, um die gesetzliche Pause nehmen zu können. Allein das ist gesetzwidrig. Gravierende Versorgungsmängel bleiben da nicht aus, ganz zu schweigen von den berufsbedingten Erkrankungen der Pflegenden, die durch schweres Heben und Tragen, Umlagern ohne Hilfe, ständigen Zeitdruck im Nacken und die übergroße Verantwortung für so viele Menschen ausgelöst werden. Es wird bei weitem nicht genug getan, um beruflich Pflegende vor gesundheitlichen Schäden zu schützen.

? Wie können die Arbeitgeber für gesündere Arbeitsbedingungen sorgen?

Knüppel: Für jeden Arbeitsplatz, gerade in der Pflege, sind Gefährdungsanalysen zwingend vorgeschrieben. Hier sollen nicht nur physische Gefährdungen, sondern auch

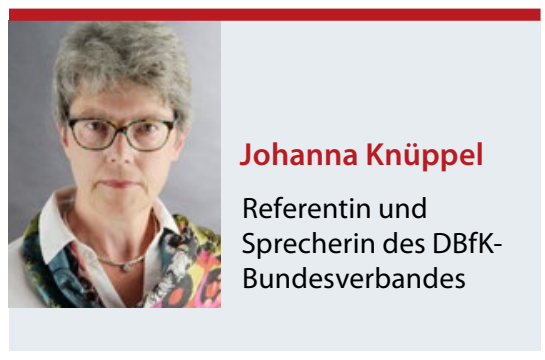

Risiken für psychische Erkrankungen erfasst, dokumentiert und entsprechend gesundheitsfördernde Maßnahmen abgeleitet werden. Allerdings: Die wenigsten Betriebe halten sich daran. Die Verpflichtung zur Gefährdungsanalyse wird flächendeckend ignoriert - und bisher überprüfen das die Unfallkassen und Berufsgenossenschaften leider auch kaum, von Sanktionen ganz zu schweigen. In Gesundheitsschutz der Beschäftigten, gesundheitsfördernde Arbeitsplatzgestaltung, Ergonomie, Prävention ... wird bisher in der Pflege in Deutschland viel zu wenig investiert. Und wenn doch, dann häufig an den Beschäftigten und deren Bedürfnissen vorbei.

\section{? Wo sehen Sie Handlungsbedarf?}

Knüppel: Pflege als Beruf ist für den potenziellen Berufsnachwuchs so unattraktiv geworden, dass immer weniger junge Menschen dort einmünden. Der hohe Altersdurchschnitt in den Pflegeberufen ist ein großes Risiko für die Versorgungssicherheit. In wenigen Jahren steht uns eine große Verrentungswelle bevor. Was dann? Es führt kein Weg daran vorbei, alles dafür zu tun, damit Pflegefachpersonen so motiviert, engagiert, gesund und so lange wie irgend möglich in ihrem Beruf arbeiten können. Hierzu haben viele Arbeitgeber wie auch die politisch Verantwortlichen ihre Hausaufgaben längst nicht gemacht!

Das Interview führte Nicoletta Eckardt. 